\title{
Application of Boltzmann's Kinetic Equation to Research of Approach to Equilibrium Thermal Radiation of Rarefied Gas
}

\author{
A. N. Volobuev \\ Samara State Medical University, Department of Physics, Samara, 443086, Russia \\ Email: volobuev47@yandex.ru
}

\begin{abstract}
On the basis of the kinetic equation for interaction of the electromagnetic radiation quantums with rarefied gas the time dynamics of approach to equilibrium thermal radiation of quantum distribution function and density of radiation energy is investigated. It is shown, that in a seen interval of electromagnetic waves this process lasts some micro seconds. The deduction of the kinetic equation of interaction of quantums with atoms of rarefied gas is submitted. Some consequences from this equation are investigated.
\end{abstract}

Keywords: Boltzmann's kinetic equation, thermal radiation, function of distribution.

\section{Introduction}

Transient processes at thermal radiation are of interest in various areas of technics and biology. Unfortunately, to calculate duration of transient radiating processes in complex, for example, organic systems, it is practically impossible. But for rather simple systems such as rarefied gas this analysis is quite feasible. It can be carried out with the help of the Boltzmann's kinetic equation.

The theories connected to the Boltzmann's kinetic equation it is rather widely applied to the analysis of various physical processes [1]. With the help of such theories interactions of the ideal gas molecules, of the particles in plasma, of the phonons in crystals, etc. have been successfully analysed. The most exact deduction of Maxwell's distribution at the ideal gas molecules in the velocities (or their kinetic energies) is carried out with the help of the Boltzmann's kinetic equation [2] at examination of cooperating atoms system in a stationary condition. The Boltzmann's kinetic equation first of all reflects non-stationary process of the particles interaction among themselves, influence of this interaction on an establishment of distribution of molecules, influence on investigated system of external factors.

The method of the kinetic equations with use of a principle of detailed balance is applied also at research of transition of the atoms cooperating with electromagnetic radiation from one state in another [2].

For photons in difference, for example, from molecules of gas the energy distribution can vary only at their interaction with atoms of substance [2]. Therefore character of transient to equilibrium thermal radiation of gas should depend on characteristics of gas, distribution of the particles directly cooperating with radiation.

In works which investigate the Boltzmann's kinetic equation for photons in gas it is possible to allocate two directions.

The first direction uses so-called the one-velocity theory of carry of particles $[3,4,5]$. The basic attention in these works is given to the kinetic equation for atoms of substance. The kinetic equation for radiation is written similarly and as a whole auxiliary character has. Transition of radiation to equilibrium distribution of Bose - Einstein usually is not analyzed.

The second direction is connected to A.S. Kompaneets' basic work [6]. The further analysis of the kinetic equation offered him is carried out in $[7,8]$. The main lack of this equation in our opinion is the assumption that function of electrons distribution is Maxwell's. But also till now this equation is used in works on scattering of photons on electrons in astrophysical objects [9]. The research submitted in the given work as a whole corresponds to the second direction.

It is possible to note also that the examined system of photons and electrons interaction in rarefied gas differs from similar system in a solid body where the accounting of the Pauli's principle (besides 
function of electrons distribution in atom) in system of electronic gas $[2,10,11]$ is necessary. It complicates integral of interaction.

The purpose of the present work is reception and the analysis of the Boltzmann's kinetic equation for system "photons - rarefied gas", research of time dynamics of approach of the photon energy distribution to equilibrium distribution of the Bose - Einstein, and the density of thermal radiation energy to equilibrium law of Stefan - Boltzmann, and deviations of non equilibrium radiation from the law of the fourth degree of temperature.

\section{The Kinetic Equation of Electromagnetic Radiation with Rarefied Gas Interaction}

The kinetic equation for radiation in the medium received by a method of multipartial function of distribution [12] hardly it is possible to assume for our purposes satisfactory. It is connected to absence of influence of radiation on distribution of the medium particles. Therefore for a finding of the Boltzmann's kinetic equation of system «photons - rarefied gas» is interest to consider in more detail the transient process of an establishment of equilibrium quantum distribution of electromagnetic radiation. For this purpose we shall consider interaction of some flux of monochromatic quantums by energy $\varepsilon=h v$ for nonequilibrium electromagnetic radiation where $h$ is a Planck's constant, $\boldsymbol{v}$ - frequency of electromagnetic radiation, with atoms of substance in some volume $V$, fig. 1 .

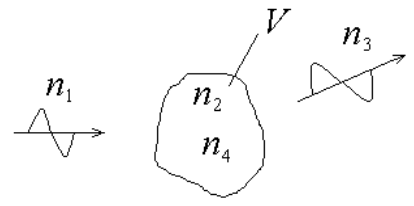

Figure 1. Interaction of quantums of electromagnetic energy with volume of rarefied gas

Number of the quantums falling on volume in unit of time we shall designate $n_{1}$, number of the quantums which are taking off from volume $V$ on all directions we shall designate $n_{3}$. System in which quantums cooperate we shall present in the following kind. We shall assume that it represents rarefied gas and will consist of identical particles - atoms which can be in two states: $n_{2}$ particles are in the first state (not excited atoms) and $n_{4}$ particles are in the second state (the excited atoms).

Change of number taken off an element of volume $d V$ photons $d n_{3}$ is determined by the following reasons: change of number of falling photons $d n_{1}$; or change of number of photons owing to their interaction with particles in the first state $d n_{2}$ (absorption of quantums by the not excited atoms of substance and their excitation), the process $n_{2} \rightarrow n_{4}$; or change of number of photons owing to transition (spontaneous or compelled) particles from the second state in the first state $d n_{4}$, the process $n_{4} \rightarrow n_{2}$ (radiation of photons from substance the excited atoms).

Hence:

$$
d n_{3}=d n_{1}-d n_{2}+d n_{4} .
$$

It is accepted that one quantum there can be only one of the listed above events.

Change of number of the quantums flying in volume $d V$ we shall present as:

$$
d n_{1}=\frac{2}{h^{3}} f_{1} d p_{1} d V,
$$

where $d p_{1}=d p_{1 X} d p_{1 Y} d p_{1 Z}$ there is product of changes a component of flying quantums impulse, and $d \gamma_{1}=d p_{1} d V$ - an element of phase volume in which quantums flying in volume $V$ get. Size $f_{1}=f_{1}\left(\mathbf{r}_{1}, \mathbf{p}_{1}, t\right)$ is a function of flying quantums distribution (density of probability for quantum to have the given characteristics: position, an impulse or energy), $\mathbf{r}_{1}$ - a radius - vector of the flying quantum, $h^{3}$ - the element of phase volume occupied by a photon (or atom), the factor 2 reflects two possible polarizations of a photon in volume $h^{3}$. 
Let's designate change of an atom impulse owing to its excitation by quantum $d p_{2}$. Obviously, under of an impulse preservation law is $d p_{2}=-d p_{1}$ (reduction of a quantum impulse results to increase of an atom impulse). Therefore in process $n_{2} \rightarrow n_{4}$ the element of phase volume $d \gamma_{2}=d p_{2} d V$ in which atoms cooperating with quantums is negative. It is possible to assume that the cooperating quantum and atom are in one point therefore $\left|d \gamma_{1}\right|=\left|d \gamma_{2}\right|$. Having divided the equations (1) on phase volumes of corresponding processes, we receive:

$$
\frac{d n_{3}}{d \gamma_{1}}-\frac{d n_{1}}{d \gamma_{1}}=\frac{d n_{2}}{d \gamma_{2}}+\frac{d n_{4}}{d \gamma_{2}}=\frac{2}{h^{3}} \theta
$$

where $\theta$ there is some value which physical sense we shall determine later. The factor $\frac{2}{h^{3}}$ is used for simplification of transformations. The size $\frac{d n_{2}}{d \gamma_{2}}$ is used with plus since for size $d n_{2}$, i.e. for process $n_{2} \rightarrow n_{4}$, the phase volume $d \gamma_{2}$ is negative.

Using (2) and (3) it is possible to write down:

Having designated:

$$
d n_{3}=\frac{2}{h^{3}}\left(\theta+f_{1}\right) d \gamma_{1}
$$

$$
d n_{4}=\frac{2}{h^{3}} f_{2} d p_{2} d V
$$

where $f_{2}=f_{2}\left(\mathbf{r}_{2}, \mathbf{p}_{2}, t\right)$ is some functional of distributions of the excited atoms (the set of the distribution functions), $\mathbf{r}_{2}$ - a radius - vector of atom radiating quantum, factor 2 - two possible orientations of an electron spin in radiating atom in volume $h^{3}$.

A functional $f_{2}$ on the one hand reflects electron distribution in atom (i.e. distribution of particles with a semi-whole spin) - internal distribution, on the other hand it reflects external thermal power distribution of atoms. Both it is distributions can change at a photon irradiation of the gas system.

From (3) and (5) we shall find:

$$
d n_{2}=\frac{2}{h^{3}}\left(\theta-f_{2}\right) d \gamma_{2} .
$$

The number of the quantum interactions which have flown in volume $d V$ with not excited atoms of substance is equal:

$$
d n_{1} d n_{2}=\frac{4}{h^{6}} f_{1}\left(\theta-f_{2}\right) d p_{1} d V d \gamma_{2} .
$$

This number of quantums gets in phase volume $d \gamma_{1} d \gamma_{2}$.

On the other hand this phase volume leaves the certain number of the quantums which have taken off from volume $V$ :

$$
d n_{3}{ }^{\prime} d n_{4}{ }^{\prime}=\frac{4}{h^{6}} f_{2}{ }^{\prime}\left(\theta+f_{1}{ }^{\prime}\right) d p_{2} d V d \gamma_{1} .
$$

The strokes at functions of distribution mean that a kind of the distribution functions both at photons, and at the excited atoms can change during time before and after interaction of quantums with atoms.

The remained in phase volume $d \gamma^{2}=d \gamma_{1} d \gamma_{2}$ quantums can be found under the formula:

$$
d^{2} n=\frac{4}{h^{6}}\left[f_{1}\left(\theta-f_{2}\right)-f_{2}{ }^{\prime}\left(\theta+f_{1}^{\prime}\right)\right] \delta\left(h v^{*}-\varepsilon_{i}^{*}\right) d \gamma^{2} .
$$

In the formula (9) $\delta\left(h v^{*}-\varepsilon_{i}^{*}\right)$ is $\delta$-function which takes into account that only at energy of quantum $h v$, corresponding energy of electronic transition $\varepsilon_{i}$, there is an interaction of quantums to atoms of substance. Asterisks at sizes mean that comparison goes on dimensionless energies the scale at which is identical. The similar approach, for example, is carried out in [1].

Let's copy the formula (9) as: 


$$
d f=d\left(\frac{h^{3}}{2} \frac{d n}{d \gamma}\right)=\frac{2}{h^{3}}\left[f_{1}\left(\theta-f_{2}\right)-f_{2}{ }^{\prime}\left(\theta+f_{1}^{\prime}\right)\right] \delta\left(h v^{*}-\varepsilon_{i}^{*}\right) d \gamma,
$$

where $f=f(\mathbf{r}, \mathbf{p}, t)$ there is the time-dependent function of the quantum distribution which before interaction had function the distribution function $f_{1}$, and after interaction $f_{1}{ }^{\prime}$. Accordingly for this time has changed and functional distributions of atoms from $f_{2}$ up to $f_{2}^{\prime}$.

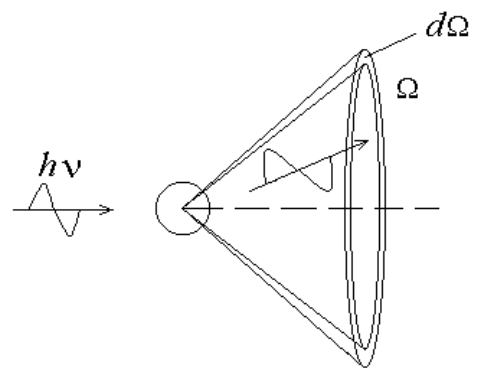

Figure 2. Interaction of quantum of electromagnetic energy with atom

Let's introduce effective section of interaction $\sigma$ of quantum with atom. Depending of effective section change of interaction on a solid angle of quantum scattering $\Omega$, fig. 2 , it is possible to write down as:

$$
d \sigma=\sigma d \Omega .
$$

Hence the volume element of interaction becomes:

$$
d V=c d \sigma=c \sigma d \Omega .
$$

In the formula (12) the unit time interval is examined, therefore instead of length of a volume element the quantum velocity $c$ is used. Besides we have neglected velocity of an atom movement in comparison with quantum velocity of electromagnetic radiation cooperating with atom.

Taking into account $|\mathbf{p}|=\frac{h v}{c}$, and $d p=d p_{X} d p_{Y} d p_{Z}$ we shall find an element of phase volume:

$$
d \gamma=d p d V=\frac{h^{3}}{c^{3}} d v^{3} \sigma c d \Omega=\frac{h^{3}}{c^{2}} \sigma d v^{3} d \Omega .
$$

Integrating the expression (10) on all frequencies and a solid angle with the account (13) we receive:

$$
\Delta f=\frac{2}{c^{2}} \iint_{v, \Omega} \sigma\left[f_{1}\left(\theta-f_{2}\right)-f_{2}{ }^{\prime}\left(\theta+f_{1}{ }^{\prime}\right)\right] \delta\left(h v^{*}-\varepsilon_{i}^{*}\right) d v^{3} d \Omega .
$$

In (14) change of distribution function $\Delta f$ in unit of time is examined, therefore in the left part (14) will be speed of change of the distribution function $\frac{d f}{d t}[1]$. In the right part (14) between functions of distribution without strokes and with strokes the time interval also is equal to unit. Hence:

$$
\frac{d f}{d t}=\frac{2}{c^{2}} \iint_{v, \Omega} \sigma\left[f_{1}\left(\theta-f_{2}\right)-f_{2}{ }^{\prime}\left(\theta+f_{1}{ }^{\prime}\right)\right] \delta\left(h v^{*}-\varepsilon_{i}^{*}\right) d v^{3} d \Omega,
$$

where $I=\frac{2}{c^{2}} \iint_{v, \Omega} \sigma\left[f_{1}\left(\theta-f_{2}\right)-f_{2}{ }^{\prime}\left(\theta+f_{1}{ }^{\prime}\right)\right] \delta\left(h v^{*}-\varepsilon_{i}^{*}\right) d v^{3} d \Omega$ there is integral of interaction of electromagnetic radiation quantums with atom of substance. The integral of the interaction similar to received is found in [1] for scattering of phonons in crystals on impurity.

The equation (15) is the kinetic equation of interaction of electromagnetic radiation quantums and atoms.

The solution of the integro-differential equation (15) rather difficult mathematical problem, therefore we shall consider two special cases. 


\section{A State of Equilibrium at Interaction of Radiation with Atoms of a Gas}

At interaction of the equilibrium electromagnetic radiation quantums and equilibrium system of atoms the distribution of quantums and atoms of substance does not change, i.e. $\frac{d f}{d t}=0$. From this condition according to (15) it is possible to write down that

$$
f_{1}\left(\theta-f_{2}\right)-f_{2}\left(\theta+f_{1}\right)=0 .
$$

The functional equation with two unknown functions which has infinite set of solutions is received. Strokes in the equation (16) are omitted since functions $f_{1}$ and $f_{2}$ in this case in due time do not change.

One of functions of distribution $f_{1}$ in the equation (16) should satisfy to distribution of Bose - Einstein for photons radiated by the absolutely black body [13]:

$$
f_{1}=\frac{1}{\mathrm{e}^{X}-1}
$$

where $X=\frac{\varepsilon_{i}-\mu}{k T}$, and $\varepsilon_{i}$ is energy of quantum in phase volume $d \gamma_{i}, \mu$ - the chemical potential attributed to one quantum, $k$ - Boltzmann's constant, $T$ - absolute temperature of examined quantum system.

Substitution (17) in (16) allows receive functional of atom distributions:

$$
f_{2}=\frac{1}{\mathrm{e}^{X}+(2 / \theta-1)} .
$$

At $\theta=1$ the functional (18) has transit in Fermi - Dirac's distribution [13]. In this case distribution of the excited atoms fully corresponds with distribution of electrons in these atoms. It means that the basic role at interaction of photons and atoms (i.e. at change of a system state) is played the excitation of atoms determined by transition of electrons on higher power levels. By interaction of atoms in rarefied gas it is neglected. The size is $X=\frac{\varepsilon_{i}-\mu}{k T}$. But as against (17) $\varepsilon_{i}$ is energy of the excited atom in phase volume $d \gamma_{i}$ corresponding to energy of excitation of electron under action of radiation quantum by energy $\varepsilon_{i}$, and $\mu$ in this case is energy of Fermi's level.

At $\theta=2$ the distribution of atoms of substance in a condition of equilibrium (18) is become identical to Maxwell's distribution. It means that the basic role at interaction of electromagnetic radiation quantums and atoms is played not excitation of atoms, but the change of their kinetic energy influencing on distribution of atoms.

Thus it is possible to draw a conclusion that size $\theta$ reflects character of interaction of quantums with atoms of substance: in particular, at $\theta=1$ the basic influence on distribution of atoms it is occurs owing to their excitation, at $\theta=2$ owing to change of their kinetic energy.

For intermediate non-equilibrium value of functional $f_{2}$, in case when at an irradiation of gas takes place comparable on energies excitation of atoms and change of their kinetic energy, hardly is possible to describe $f_{2}$ the uniform formula such as (18).

The kinetic equation (15) can be copied using expression for a full derivative in the left part:

$$
\frac{\partial f}{\partial t}+c \frac{\partial f}{\partial r}+F_{c} \frac{\partial f}{\partial p}=\frac{2}{c^{2}} \iint_{\nu, \Omega} \sigma\left[f_{1}\left(\theta-f_{2}\right)-f_{2}{ }^{\prime}\left(\theta+f_{1}{ }^{\prime}\right)\right] \delta\left(h v^{*}-\varepsilon_{i}^{*}\right) d v^{3} d \Omega
$$

where $F_{c}=\frac{\partial p}{\partial t}$ is the force working on a photon, for example, with external gravitational field [14].

\section{The Time Dynamics of Transition of Radiation in an Equilibrium Condition}

Let's consider time asymptotics of the function $f_{1}$ of photon energy distribution which approach to equilibrium radiation of absolutely black body.

Let's assume that functional of the atom distributions it is constant in time and represents equilibrium function $f_{2}$, so $f_{2}=f_{2}$ '. The function of photon distribution we shall present as: 


$$
f_{1}^{\prime}=f_{1}+\delta f_{1}
$$

where $f_{1}$ there is equilibrium value of function, $\delta f_{1}$ - small change of function $f_{1}$ for small time $\delta t$. In the formula (20) the first term in the right part does not depend on time, therefore $\frac{d f}{d t}=\frac{\partial f}{\partial t} \approx \frac{\delta f_{1}}{\delta t}$. Substituting (20) in (15) we shall receive:

$$
\frac{\delta f_{1}}{\delta t}=\frac{2}{c^{2}} \iint_{v, \Omega} \sigma\left[f_{1}\left(\theta-f_{2}\right)-f_{2}\left(\theta+f_{1}\right)-f_{2} \delta f_{1}\right] d v^{3} d \Omega .
$$

We assume also that distinction in energies of electronic levels is insignificant that is correct for external electrons of atom. Therefore the power electrons spectrum (as well as a spectrum of atom speeds) is assumed in the given approximation continuous and $\delta$-function in (21) is not taken into account.

The first difference in integral of interaction in the right part (21) is equal to zero identically in condition of equilibrium hence we receive:

$$
\frac{\delta f_{1}}{\delta t}=\frac{2}{c^{2}} \iint_{v, \Omega} \sigma\left[-f_{2} \delta f_{1}\right] d v^{3} d \Omega=-\frac{2}{c^{2}} \iint_{v, \Omega} \sigma f_{2} \frac{\delta f_{1}}{\delta t} \delta t d v^{3} d \Omega .
$$

Let's designate $\frac{\delta f_{1}}{\delta t}=F$. Besides we shall assume that dispersion of photons after interaction with atoms occurs uniformly in all directions, i.e. the section of interaction $\sigma$ does not depend on an angle of dispersion. Then integrating on solid angle $\Omega$ we have

$$
F=-\frac{8 \pi}{c^{2}} \int_{0}^{v} \sigma f_{2} F \delta t d v^{3} .
$$

The integrated equation which can be solved concerning function $F$ is received. Finding a third derivative from the left and right parts (23) on frequency $v$, we receive:

$$
\frac{\partial^{3} F}{\partial v^{3}}=-\frac{8 \pi}{c^{2}} \sigma f_{2} F \delta t
$$

The solution of the equation (24) we shall write down as:

$$
F=F_{0} \exp (-\alpha \sqrt[3]{\delta t})
$$

where $F_{0}$ there is a constant of integration, and size $\alpha=2 v\left(\frac{\pi}{c^{2}} \sigma f_{2}\right)^{1 / 3}$.

Taking into account $\frac{\delta f_{1}}{\delta t}=F$ we shall find:

$$
\delta f_{1}=F_{0} \delta t \exp (-\alpha \sqrt[3]{\delta t})
$$

The size $\delta t=t-t_{0}$ is a time from the initial moment $t_{0}$. If to accept $t_{0}=0$, and $f_{10}$ - the distribution of Bose - Einstein, so $\delta f_{1}=f_{1}-f_{10}$ the achievement by distribution $f_{1}$ of the equilibrium condition can be calculated under the formula:

$$
f_{1}=f_{10}+F_{0} t \exp (-\alpha \sqrt[3]{t})
$$

In the formula $\delta f_{1}=f_{1}-f_{10}$ it is carried out obvious re-designation sizes to exclude strokes.

The size $F_{0}<0$ as the probability of an equilibrium state of the quantum energy distribution is more than nonequilibrium, hence $f_{1}<f_{10}$. Therefore there is $\delta f_{1}=f_{1}-f_{10}<0$.

The formula (27) shows how nonequilibrium distribution of photon energy has approaching to equilibrium of Bose-Einstein's distribution $f_{10}$ of absolutely black body radiation. It actually gives as a first approximation the time non-stationary amendment to this distribution. At time $t$ aspiring to infinity from (27) naturally follows $f_{1}=f_{10}$. 


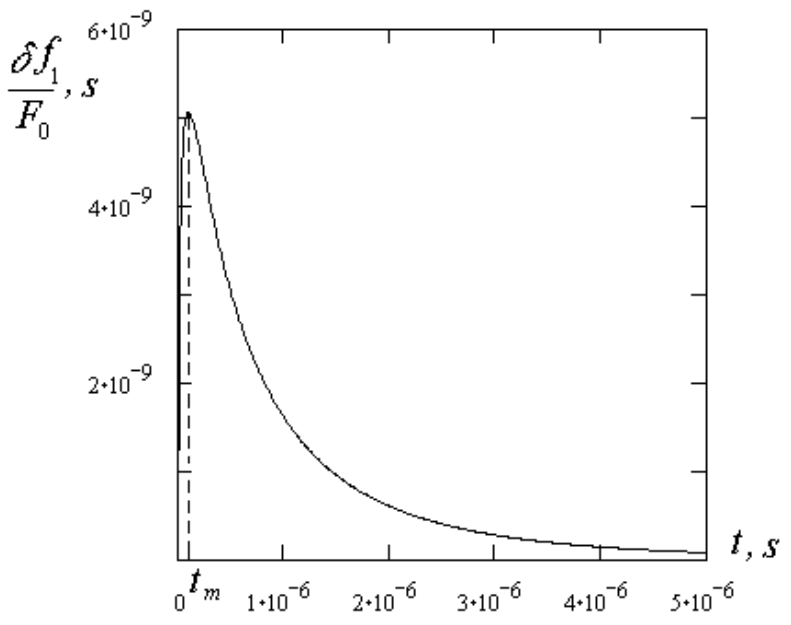

Figure 3. Time dependence of transition of quantum distribution function of thermal radiation in an equilibrium state

On fig. 3 the dependence $\frac{\delta f_{1}}{F_{0}}$ as function of time $t$ is shown. The ratio $\frac{\delta f_{1}}{F_{0}}>0$ as in numerator and a denominator are negative sizes.

Investigating on an extremum the formula $(27)$ it is received that function $\frac{\delta f_{1}}{F_{0}}$ attains a maximum at the moment of time $t_{m}=\frac{27}{\alpha^{3}}$. The descending part of the curve at $t>t_{m}$ has physical sense only. It is consequence of that in due time the size $\delta f_{1}$ should decrease on the module up to zero.

For the analysis of the function $f_{1}$ behaviour in the domain of far from equilibrium, i.e. at big on the module $\delta f_{1}$ the solution of the full kinetic equation (19) is necessary that is beyond frame of given article.

Let's estimate size of time $t_{m}$, from which the law (27) for frequency $v=0.6 \cdot 10^{15} \mathrm{~Hz}$ in a seen range of the wave lengths is correct. We shall suppose $f_{2}=0.5$. The effective section of interaction $\sigma$ can be less sizes of atom (owing to tunnel effect) or more sizes of atom (antistock's interaction). For estimation of $\sigma$ we shall assume that the effective section of interaction is approximately equal to the sizes of hydrogen atom. Hence $\sigma=0.878 \cdot 10^{-20} \mathrm{~m}^{2}$. In result we shall find parameter $\alpha=642 \mathrm{~s}^{-1 / 3}$ and time $t_{m}=10^{-7} \mathrm{~s}$. For these parameters the curve on fig. 3 is plotted.

Is interest for finding of the non-stationary amendment to the Stefan - Boltzmann's law for volumetric density of energy of thermal radiation $u$.

Using the known formula [2] after simple transformations (for photons $\mu=0$ [13]) with use (27), we shall find:

$$
u=\int_{0}^{\infty} \frac{8 \pi h}{c^{3}} f_{1} v^{3} d v=\sigma_{c} T^{4}+\frac{\beta(T)}{\sqrt[3]{t}}
$$

where $\sigma_{c}$ there is constant of Stefan - Boltzmann's. The size $\beta(T)=\frac{3 \pi h F_{0}}{c^{3}\left(\pi \sigma f_{2} / c^{2}\right)^{4 / 3}}$ depends on temperature since on temperature depends functional $f_{2}(18)$. At deduction the formula $\int_{0}^{\infty} x^{3} e^{-m x} d x=\frac{6}{m^{4}}$ is used. The formula (28) is correct for time $t>t_{m}=\frac{27}{\alpha^{3}}$.

The size is $\beta(T)<0$ owing to negative size $F_{0}$. It reflects the known fact that the volumetric density of equilibrium radiation energy of absolutely black body is greatest possible.

In due course at transition of system in an equilibrium state at $t \rightarrow \infty$ the non-stationary amendment in (28) to aspire to zero and volumetric density of radiation energy to Stefan - Boltzmann's law. With growth of temperature the contribution of the non-stationary amendment also is reduced. If to believe 
size $F_{0}$ is constant that in a case of Maxwell's distribution of molecules the size $\beta \rightarrow \frac{3 \pi h F_{0}}{c^{3}\left(\pi \sigma / c^{2}\right)^{4 / 3}}$, in case of Fermi - Dirac's distribution $\beta \rightarrow \frac{6 h F_{0}}{\left(0.5 \pi c \sigma^{4}\right)^{1 / 3}}$.

\section{Conclusion}

On the basis of Boltzmann's kinetic equation the process of interaction of rarefied gas with quantums of electromagnetic radiation is considered. The modified kinetic equation for this process is found.

Solutions of the received kinetic equation in a stationary case are investigated and in case of a small deviation of quantum distribution from stationary Bose - Einstein's distribution. It is shown that in a stationary variant to the kinetic equation satisfy distributions of Bose - Einstein for quantums and Fermi - Dirac or Maxwell for atoms of gas. Research of the solution of the kinetic equation for transient process at a small deviation of quantum distribution from equilibrium state has allowed to receive the law of approach of thermal radiation to an equilibrium state. This process proceeds some micro seconds.

The non-stationary additive to density of an energy flux in the Stefan - Boltzmann's law dependent on temperature is found.

\section{References}

1. E.M. Lifshits, L.P. Pitaevskij. "Physical kinetics." Moscow.Fizmatlit, 2002. 536 p.

2. V.G. Levich. "Course of Theoretical Physics." V. 1. Moscow. Fizmatgiz, 1962, 696 p.

3. V.S. Vladimirov. "Mathematical Problems of the One-velocity Theory of Particle Carry." Transactions of mathematical institute AN SSSR, 1961, V. 61. Pp. 3-11.

4. A.E. Bulishev, N.G. Preobrazhenskii, A.E. Suvorov. "Radiation transport in spectral lines." Sov. Phys. Usp., 1988, V. 31. P. 865-878.

5. V.E. Troshchiev, A.V. Nifanova, Iu.V. Troshchiev. "Characteristic Approach to Approximation of Conservation Law in the Kinetic Equations of Radiation Translation." DAN RF, 2004, V. 394, No 4. Pp. $454-458$.

6. A.S. Kompaneets. "Physico-Chemical and Relativistic Gaso-Dynamics." Moscow. Science, 1977, 287 p.

7. Ia.B. Zeldovich, E.V. Levich. "Bose condensation and shock waves in photon spectra." JETP, 1969, V. 28 , No. 6. P. $1287-1290$.

8. A.E. Dubinov. "Exact stationary solution of the Kompaneets kinetic equation." Tech. Phys. Lett., 2009, V. 35. P. $260-262$.

9. D.I. Nagirner, V.M. Loskutov, S.I. Grachev. "Exact and Numerical Solutions of the Kompaneets' Equation: Evolution of a Spectrum and Average Frequencies." Astrophysics, 1997, V. 40, No 3. Pp. 349 - 364.

10. O. Madelung. "Theory of Solid Body." Moscow. Science, 1980. 418 p.

11. R.Kh. Amirov, V.N. Gusyatnikov. "A dynamical influence of the constant electric field on the kinetics of photons interacting with semiconductor electrons." Semiconductors/Physics of the Solid State, 2001, V. 35, № 5. P. $528-533$.

12. A.I. Akhiezer, S.V. Peletmiski. "Methods of Statistical Physics." Moscow. Science, 1977. 368 p.

13. A.N. Matveev. "Molecular Physics." Moscow. Higher School, 1981. 400 p.

14. Ia.B. Zeldovich, I.D. Novikov. "Theory of Gravitation and Evolution of Stars." Moscow. Science, 1971. 484 p. 